\title{
Generation of the Higgs Condensate and Its Decay after Inflation
}

\author{
Kari Enqvist, Tuukka Meriniemi and Sami Nurmi \\ University of Helsinki and Helsinki Institute of Physics, P.O. Box 64, FI-00014, \\ Helsinki, Finland
}

\begin{abstract}
We investigate the dynamics of the Standard Model higgs with a minimal coupling to gravity during and after inflation. In the regime where the Standard Model vacuum is stable, we find that the higgs becomes a light spectator field after about 30 efolds of inflation, irrespectively of its initial value. Once the higgs has become light, its root-mean-square value $h_{*}$ relaxes to equilibrium in about 85 efolds for the inflationary scale of $H_{*}=10^{4} \mathrm{GeV}$ and in 20 efolds for $H_{*}=10^{10} \mathrm{GeV}$. The equilibrium value is given by $h_{*} \sim 0.36 \lambda_{*}^{-1 / 4} H_{*}$, where $\lambda_{*}=0.09 \ldots 0.0005$ is the higgs self coupling at the scales $H_{*}=10^{4} \ldots 10^{10} \mathrm{GeV}$. We show that the main decay channel of the higgs condensate after inflation is the resonant production of Standard Model gauge bosons. For a set of parameters we find that a significant part of the condensate has decayed in between 340 and 630 Hubble times after the onset of higgs oscillations, depending on $H_{*}$ in a non-trivial way. The higgs perturbations correspond to isocurvature modes during inflation but they could generate significant adiabatic perturbations at a later stage for example through a modulation of the reheating stage. However, this requires that the inflaton(s) decay no later than a few hundred Hubble times after the onset of higgs oscillations.
\end{abstract}




\section{Contents}

1. Introduction 1

2. Dynamics of the higgs field 3

2.1 During inflation 3

2.2 After inflation 5

3. Perturbative decay 6

3.1 Higgs decay into weak gauge bosons 6

$\begin{array}{lll}3.2 & \text { Higgs decay into fermions } & 7\end{array}$

$\begin{array}{lll}3.3 & \text { Higgs decay into photons } & 7\end{array}$

3.4 Higgs decay into gluons 8

4. Non-perturbative decay 8

4.1 Resonant production of gauge bosons and higgs particles 9

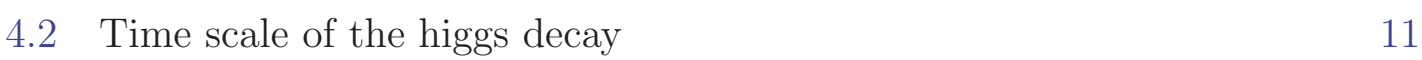

$\begin{array}{lll}4.3 & \text { Resonant production of quarks and leptons } & 14\end{array}$

5. Discussion

\section{Introduction}

The exact mechanism for inflation is not known, although a consistent description is obtained by assuming a slowly rolling scalar field, the inflaton. Since the discovery of the higgs at LHC [1], we know that there exists at least one other scalar field, which during inflation appears to be a spectator field with no dynamical role. While the higgs could in principle also act as the inflaton, this possibility is not generic but requires a strong non-minimal coupling to gravity [2] and has also been argued to have unitarity problems [3]. Although the spectator fields are insignificant during inflation, they may become important at a later stage. For instance, they could contribute to the generation of the primordial curvature perturbation, as in the curvaton scenario [4]. Their post-inflationary dynamics could also be interesting on its own right.

If the Standard Model higgs is a light field during inflation, it is also subject to fluctuations with an almost scale invariant spectrum. These fluctuations may contribute to metric perturbations in several ways. For instance, the higgs could act 
as a curvaton although the viability of this mechanism is hampered by the quartic form of the potential [5], however see also [6]. Another possibility would be that the higgs is coupled to the inflaton and generates perturbations by modulating the end of inflation [7], or the reheating process [8]. Indeed, it has been argued [9] that the entire origin of the observed primordial perturbations could be due to inflationary fluctuations of the higgs modulating the reheating process. However, as the result of the quartic self-interaction of the higgs field, the perturbations generated by this mechanism typically have significant non-Gaussian features. With the Planck limit of $f_{\mathrm{NL}}=2.7 \pm 5.8$ [10], these constrain the range of feasible inflationary scales from the below $[11,5]$.

Independently of these possibilities, it is of interest to find out what actually happens to the higgs condensate generated by the inflationary fluctuations. This is an issue that might have consequences also for the electroweak phase transition in the early universe.

While inflation lasts, the higgs generically is in slow roll, as we will discuss below. After the end of inflation and onset of inflaton oscillations, also the higgs field begins to oscillate in its potential once its effective mass roughly equals the Hubble rate. Because its energy density is subdominant, these oscillations take place in the background determined by the inflaton dynamics. As the higgs oscillates, it eventually starts to decay to other Standard Model particles it couples to. The decay could be either perturbative or non-perturbative. If the higgs field were to decay before the inflaton, there would be no possibility for generating perturbations through its modulation of the inflaton decay.

The dynamical evolution of the higgs field and its decay after inflation is a general problem which we address in this paper by analysing both the perturbative and non-perturtubative decay processes. Requiring that the higgs decays after the inflaton, which would be a necessary condition for it to source the generation of primordial perturbations through the modulated reheating, turns out to be a nontrivial constraint for two reasons: the higgs field starts to oscillate relatively soon after the end of inflation, and its couplings to other Standard Model fields are large.

The paper is organized as follows. In Sect. 2 we address the dynamics of the higgs field first during inflation, when its root-mean-square value is evolving towards the equilibrium, and then discuss the motion of the condensate after inflation. In Sect. 3 we study the perturbative decay of the higgs condensate, discussing blocking factors and computing the appropriate decay widths. In Sect. 4 we investigate the non-perturbative decay and show that the main decay process of the higgs condensate is the resonant production of weak gauge bosons. We estimate the time scale when the backreaction kicks in and the fraction of energy transferred to the decay products becomes significant. Sect. 5 summarizes the reults and contains a discussion about the higgs modulating the inflaton decay. 


\section{Dynamics of the higgs field}

\subsection{During inflation}

We consider the dynamics of the Standard Model higgs assuming the Standard Model is valid up to the inflationary energy scale, characterized by the Hubble rate $H_{*}$. We also assume that any non-minimal coupling between the higgs field and gravity can be neglected. For large field values, $h \gg v=246 \mathrm{GeV}$, the bare higgs mass is negligible compared to the quartic self-interaction term and the effective higgs potential is well approximated by

$$
V(h)=\frac{\lambda(\mu)}{4} h^{4}
$$

Here $\lambda$ is the running self-coupling and we set the renormalization $\mu$ equal to the higgs amplitude during inflation, $\mu \sim h_{*}$. This choice serves to minimize radiative corrections of the form $\ln (h / \mu)$ to the effective higgs potential. The dependence of the effective potential on $\mu$ is spurious and the choice $\mu=h_{*}$ is simply made for computational convenience. As we will discuss below, the typical higgs values during inflation lie somewhat below the inflationary scale $H_{*}$, so that we also have $\mu=h_{*} \lesssim H_{*}$.

With this framework, the running of the coupling $\lambda(\mu)$, and thereby the higgs potential, is fully determined and has been computed up to three-loop precision [12]. The coupling decreases towards larger energies and eventually hits zero $\lambda\left(\mu_{\text {inst }}\right)=0$ at an instability scale $\mu_{\text {inst }}$. For the best fit values of Standard Model parameters the instability scale lies around $\mu_{\text {inst }} \sim 10^{11} \mathrm{GeV}$ and is pushed up to $\mu_{\text {inst }} \sim 10^{15} \mathrm{GeV}$ by decreasing the top mass to $m_{t}=171.0 \mathrm{GeV}, 3-\sigma$ below the best fit value [12]. At energies above the instability scale, the coupling $\lambda$ becomes negative implying an instability of the Standard Model vacuum. Here we require the higgs potential remains stable during inflation $\lambda(\mu)>0$. This bounds the inflationary scale $H_{*}$ and the higgs value $h_{*}$ to lie below the instability scale $\mu_{\text {inst }}$. The coupling is bounded by $\lambda \lesssim 0.1$ for field values $h_{*} \gtrsim 10^{2} \mathrm{GeV}$ and by $\lambda \lesssim 0.01$ for $h_{*} \gtrsim 10^{8} \mathrm{GeV}$ [12]. The higgs mass at inflationary scales is then $V^{\prime \prime}\left(H_{*}\right) \gg(125 \mathrm{GeV})^{2}$, unless $h_{*} \simeq \mu_{\text {inst }}$, and ignoring the bare mass in (2.1) is indeed well justified.

While the higgs potential is fully determined the initial field value at the onset of inflation is an a priori unknown parameter. For large initial values $h \gtrsim H / \sqrt{\lambda}$ the field is effectively massive and oscillates around the minimum of its potential. The amplitude decreases exponentially during inflation, $h \sim e^{-N}$. The field enters very fast the regime $h \ll H / \sqrt{\lambda}$ where its effective mass is small compared to the Hubble rate. Even if the higgs started with an initial value of $h \sim M_{P}$ (which would imply a very low top mass to keep the vacuum stable) this would take only about 32 (18) efolds for the inflationary scale $H_{*}=10^{4} \mathrm{GeV}\left(H_{*}=10^{10} \mathrm{GeV}\right)$. The higgs field will then slowly evolve towards the minimum of its potential following the classical slow roll dynamics until the classical drift has decreased comparable to the source term 
from quantum fluctuations at around $V^{\prime} \sim H^{3}$. From this point on, the quantum fluctuations of the higgs field stretched to superhorizon scales effectively make the field amplitude to perform a random walk.

Using the stochastic approach [13], one may obtain an equilibrium probability distribution for the mean field value $h_{*}$ during inflation. It is given by

$$
P(h)=C \exp \left(-\frac{2 \pi^{2} \lambda_{*} h_{*}^{4}}{3 H_{*}^{4}}\right),
$$

where $C$ is a normalization constant.

Strictly speaking, the equilibrium distribution is an asymptotical state valid only after an infinity of efolds. Following the evolution of the probability distribution from an initial state peaked around some field value, one observes the distribution both spreading out approaching the equilibrium form and its central value moving towards the equilibrium result. For a quartic potential, the spreading of the probability distribution is characterized by a decoherence time (the time scale for the approach of the variance to the equilibrium result) which in terms of efolds has been found [14] to be given by $N_{\mathrm{dec}} \approx 6 \lambda^{-1 / 2}$. For $\lambda \gtrsim 0.005$, which corresponds to an inflationary scale of $H_{*}=10^{10} \mathrm{GeV}$, this yields $N_{\mathrm{dec}} \approx 85$. For comparison, for the low inflationary scale of $H_{*}=10^{4} \mathrm{GeV}$ one would find $\lambda \gtrsim 0.09$ and equilibration at $N_{\text {dec }} \approx 20$. The relaxation time, measuring the rate of approach of the central value of the distribution towards the equilibrium result, is bigger by roughly a factor of two [14]. Therefore, it appears quite natural to assume that the higgs fluctuations on observable scales follow the equilibrium distribution, provided that the horizon crossing of the largest observable scales was preceded by a few tens of extra e-foldings or more.

Making this relatively mild assumption, we may thus estimate the typical field value and the effective higgs mass at the horizon crossing of observable scales by the expectation values computed from the distribution (2.2). For the higgs mass we then find the result

$$
m_{h_{*}}^{2}=3 \lambda\left\langle h_{*}^{2}\right\rangle \simeq 0.40 \lambda_{*}^{1 / 2} H_{*}^{2},
$$

indicating that the higgs is a light field during inflating, $\eta_{h}=V_{h h} /\left(3 H^{2}\right) \lesssim \mathcal{O}(0.01)$.

The expectation value of the higgs amplitude over the entire inflating patch is vanishing $\left\langle h_{*}\right\rangle=0$ as a result of the symmetric potential. However, the variance is non-zero and the typical higgs amplitude in a random patch of the size of our observable universe is given by the root mean square value

$$
h_{*} \sim \sqrt{\left\langle h_{*}^{2}\right\rangle} \simeq 0.36 \lambda_{*}^{-1 / 4} H_{*} .
$$

Computing the corresponding energy density we find that the higgs condensate constitutes only a tiny fraction of the total energy density during inflation, $\rho_{h} / \rho_{\text {tot. }} \sim$ $10^{-3}\left(H_{*} / M_{\mathrm{P}}\right)^{2}$. We reiterate that these results are generic predictions for the Standard Model higgs assuming the Standard Model remains as a valid description and its vacuum stable up to inflationary energy scales. 


\subsection{After inflation}

The actual mass of the higgs field in our observable patch during inflation may of course deviate from the ensemble average (2.3) as a result of a statistical fluke. However, regions where the higgs mass significantly differs from $m_{h_{*}}^{2}=\mathcal{O}(0.1) \lambda_{*}^{1 / 2} H_{*}^{2}$ after a few tens of e-folds of inflation occur with suppressed probabilities. Using the equilibrium distribution (2.2) we find that the probability to obtain field values $h_{*}$ corresponding to $\eta_{h}>0.1$ (i.e. $m_{h_{*}}^{2}>0.1 \times 3 H_{*}^{2}$ ) is as low as $10^{-4}$ for $\lambda_{*}=0.01$ and it decreases exponentially for larger effective higgs masses. This simple line of reasoning suggests that the Standard Model higgs generally is a light spectator field during inflation. The inflationary stage therefore gives rise to a nearly scale invariant perturbations of the higgs field.

Due to the tiny energy density of the higgs condensate, its perturbations amount to isocurvature fluctuations during inflation which have little impact on the adiabatic metric fluctuations under this epoch. Depending on the couplings between the higgs field and the beyond Standard Model physics that drives the inflationary stage, the higgs perturbations can however be converted into observable metric perturbations after the end of inflation. This would happen if the expansion history is sensitive to the exact value of the higgs condensate, and its slight variation on superhorizon scales, as for example in the modulated reheating scenario [8] and the curvaton scenario [4].

A necessary condition for both scenarios is that the inflaton decay and reheating of the universe occurs before the decay of the higgs condensate. The decay rate of higgs into other Standard Model fields is fully determined by the measured Standard Model parameters. We can therefore unambiguously compute the decay time of the higgs condensate ${ }^{1}$. If the inflaton field decays before this epoch the Standard Model higgs with a minimal coupling to gravity could generate significant primordial perturbations through a modulation of the reheating stage. However, if the inflaton decay occurs at a significantly later stage the higgs fluctuations cannot be converted into adiabatic perturbations by such a mechanism.

To discuss the higgs dynamics after the end of inflation we assume for definiteness that the inflaton field, or the scalar parameterizing the adiabatic direction at this stage, ends up oscillating in a quadratic potential

$$
V(\phi)=\frac{1}{2} m_{\phi}^{2} \phi^{2} .
$$

The universe is then effectively matter dominated $H \propto a^{-3 / 2}$. In the following we will approximate the Hubble rate at the end of inflation by its value at the horizon crossing of observable scales, $H_{\text {end }}=H_{*}$.

\footnotetext{
${ }^{1}$ We ignore the decay channels of the higgs into the beyond Standard Model inflaton(s). This could imply non-trivial constraints on the inflaton sector and we plan to address these in a future work.
} 
After the end of inflation the higgs dynamics is classical and the field starts to roll down from $h_{*}$ towards the minimum of the potential. Soon after the end of inflation the scaling of the higgs amplitude is given by $h \propto a^{-1}$, neglecting the logarithmic running of the coupling $\lambda(h)$. Using (2.3) we then find that higgs becomes effectively massive, $m_{h}^{2} \sim H_{\text {osc }}^{2}$, at

$$
\frac{H_{\mathrm{osc}}}{H_{*}} \sim \frac{1}{4} \lambda_{*}^{3 / 4}
$$

which corresponds to $t_{\mathrm{osc}} \sim 3 \lambda_{*}^{-3 / 4} H_{*}^{-1}$ Hubble times after the end of inflation. Putting in numbers, for $\lambda_{*} \gtrsim \mathcal{O}(0.01)$ this gives $t_{\text {osc }} \lesssim \mathcal{O}\left(10^{2}\right) H_{*}^{-1}$, or equivalently $n_{\phi} \lesssim \mathcal{O}(10)$ inflaton oscillations. The higgs oscillations therefore start relatively soon after the end of inflation. Even if the inflaton would decay before higgs, the higgs value at the decay time then in general differs from the value at the time of inflation $h\left(t_{\mathrm{dec}}\right) \neq h_{*}$.

In the following we will systematically analyze the decay channels of the oscillating Standard Model higgs condensate. Identifying the dominant decay channels we will work out the time when it decays and compare it to the number of inflation oscillations.

\section{Perturbative decay}

The Standard Model higgs could decay perturbatively into quarks, leptons and gauge fields. However, it turns out that the decay channels into weak gauge bosons $h \rightarrow W W, h \rightarrow Z Z$ and top quarks $h \rightarrow t t$ that could lead to an efficient perturbative decay are kinematically blocked due to masses generated by the large higgs amplitude. The kinematically allowed perturbative decay channels on the other hand lead to a very inefficient decay.

\subsection{Higgs decay into weak gauge bosons}

The effective masses of the weak gauge bosons generated by the higgs expectation value are given by

$$
m_{W}=\frac{g h}{2}, \quad m_{Z}=\sqrt{g^{2}+g^{\prime 2}} \frac{h}{2}>m_{W} .
$$

These channels are kinematically blocked if $m_{h}<2 m_{W}$ which corresponds to $\lambda(\mu)<$ $g^{2}(\mu) / 3$. Setting $\mu \sim h$ this translates into $h \gtrsim 10^{2} \mathrm{GeV}$ so that for these higgs values there is no phase space available for the decays $h \rightarrow W W, h \rightarrow Z Z$. Using equation (2.3) we find that it takes about

$$
t \sim \lambda\left(H_{*}\right)^{-3 / 8}\left(\frac{H_{*}}{10^{2} \mathrm{GeV}}\right)^{3 / 2} H_{*}^{-1}
$$


Hubble times after the end of inflation until the higgs amplitude has decreased from the inflationary value (2.4) to the threshold $h \sim 10^{2} \mathrm{GeV}$ at which the perturbative decay into gauge bosons becomes possible. For $H_{*} \gtrsim 10^{5} \mathrm{GeV}$ this corresponds to, $t \gtrsim 10^{6} H_{*}^{-1}$ Hubble times, or equivalently $n_{\phi} \gtrsim 10^{5}$ inflaton oscillations. The perturbative higgs decay into the weak gauge bosons therefore remains blocked for a long time after the end of inflation unless the inflationary scale would be extremely low, $H_{*} \ll 10^{5} \mathrm{GeV}$.

\subsection{Higgs decay into fermions}

The masses of quarks and leptons are given by

$$
m_{i}(h)=\frac{y_{i}(h)}{\sqrt{2}} h
$$

where $y_{i}(h)$ denote the Yukawa couplings at the energy scale set by the higgs value $h$. For the Standard Model higgs the perturbative decay into top quarks $h \rightarrow \bar{t} t$ is kinematically blocked. All other fermionic decay channels are allowed unless the higgs value $h$ is tuned extremely close to the instability scale $\lambda\left(h_{\text {inst }}\right)=0$.

To estimate the time scale of the higgs decay into fermions it suffices to consider decays into bottom quarks. All other kinematically allowed fermionic decay channels are suppressed by the small Yukawas and their impact can be neglected here. The decay rate of the process $h \rightarrow \bar{b} b$ for the higgs value $h_{\text {osc }}$ corresponding the onset of oscillations is given by [15]

$$
\Gamma(h \rightarrow b b)=\frac{3 \sqrt{3 \lambda} y_{b}^{2} h_{\mathrm{osc}}}{16 \pi}\left(1-\frac{2 y_{b}^{2}}{3 \lambda}\right)^{3 / 2} \sim 10^{-6} \lambda_{*}^{3 / 4} H_{*} .
$$

In the last step we have used that $y_{b}=\mathcal{O}\left(10^{-2}\right)[12]$ and $h_{\mathrm{osc}} \sim 0.1 \lambda_{*}^{1 / 4} H_{*}$ which follows from equations (2.3) and (2.6). Setting $H=\Gamma(h \rightarrow b b)$ we find that it takes $t \gg 10^{6} H_{*}^{-1}$ Hubble times, or $n_{\phi} \gg 10^{5}$ inflaton oscillations, until the higgs can decay perturbatively to bottom quarks. Decays into lighter quarks and fermions would occur even later due to the smaller couplings.

\subsection{Higgs decay into photons}

The Standard Model higgs also decays into photons through loop mediated processes. For $h \rightarrow \gamma \gamma$ the dominant contributions come from the loops containing W-bosons and top quarks. As we have noted above, these have masses much higher than higgs, $m_{t}, m_{W} \gg m_{h}$, during the epoch we are considering. In this limit, the decay rate of the higgs into two photons at the onset of oscillations is given by [15]

$$
\Gamma(h \rightarrow \gamma \gamma) \sim 0.02 \alpha^{2} \lambda_{*}^{3 / 2} h_{\mathrm{osc}} .
$$

The fine structure constant $\alpha=g^{2} g^{\prime 2}\left(4 \pi\left(g^{2}+g^{\prime 2}\right)\right)^{-1}$ is small, $\alpha \lesssim 0.02$, in the entire regime where the Standard Model vacuum is stable [12]. From equations (2.4) and 
(2.6) we obtain $h_{\mathrm{osc}} \sim 0.1 \lambda_{*}^{1 / 4} H_{*}$. Comparing equations (3.5) and (3.4), we then immediately find that $\Gamma(h \rightarrow \gamma \gamma) \ll \Gamma(h \rightarrow b b)$. Hence the decay channels into photons are always insignificant compared to the higgs decay into bottom quarks .

The same holds true for the higgs decay $h \rightarrow \gamma Z$ mediated by quark and Wboson loops. This decay channel becomes kinematically allowed for $h \lesssim 10^{6} \mathrm{GeV}$ as $m_{h}<m_{Z}[12]$. The decay rate is approximately given by [15]

$$
\Gamma(h \rightarrow Z \gamma) \sim 10^{-5} g^{2} \lambda^{3 / 2} h_{\mathrm{osc}} .
$$

Using that $h \leq h_{\mathrm{osc}} \sim 0.1 \lambda_{*}^{1 / 4} H_{*}$ as the decay channel opens and comparing with (3.4) we find that $\Gamma(h \rightarrow Z \gamma) \ll \Gamma(h \rightarrow b b)$. These decay channels are therefore also insignificant.

\subsection{Higgs decay into gluons}

The higgs decay to two gluons is mediated by quark loops. The largest contribution comes from the top quarks. The decay rate at the onset of higgs oscillations is given by $[15]$

$$
\Gamma(h \rightarrow g g) \sim 10^{-5} g_{s}^{4} \lambda^{3 / 2} h_{\mathrm{osc}} .
$$

The factor $g_{s}^{4}$ comes from the two verteices which connects gluons to a top quark loop. Using that $h_{\mathrm{osc}} \sim 0.1 \lambda_{*}^{1 / 4} H_{*}$ and comparing with (3.4) we clearly see that $\Gamma(h \rightarrow g g) \ll \Gamma(h \rightarrow b b)$ and the decay into gluons is irrelevant compared to the decay channel into bottom quarks.

\section{Non-perturbative decay}

In the previous section we have demonstrated that perturbative decay of the Standard Model higgs becomes efficient only after a long period of Hubble times. This is due to the kinematical blocking of the decay channels $h \rightarrow W W, Z Z, t t$ with the largest couplings.

The kinematical blocking is however instantaneously removed each time when the oscillating higgs field crosses zero and the masses induced by the higgs amplitude vanish. During these short time intervals the Standard Model higgs can decay non-perturbatively into gauge bosons and fermions. The non-perturbative decay of a generic oscillating scalar field after inflation has been extensively studied in the literature, see e.g. [16, 17, 18]. The non-perturbative decay occurs much faster than the perturbative decay and it turns out to be the dominant decay channel of the higgs condensate, similar to what has been found in the context of higgs inflation [19].

Irrespectively of the type of decay products, the higgs fluctuations cannot source the generation of adiabatic metric perturbations through a modulation of the reheating stage after the higgs condensate has decayed. Therefore, one of our goals here is 
to identify the time scale of the non-perturbative higgs decay which places a lower limit for the inflaton decay rate in a successful modulated reheating scenario with the Standard Model higgs.

We will investigate both the bosonic and fermionic decay channels, including the decay of the higgs condensate into higgs particles. In our analysis we will neglect the backreaction of the resonantly produced particles to the higgs dynamics, which eventually shuts down the resonance. We will also neglect the perturbative decays of the produced particles into other Standard Model fields. This is justified as the perturbative decay widths are small compared to the Hubble rate. For example, the decay width of the $\mathrm{W}$-bosons to leptons and quarks at the onset of higgs oscillations is given by

$$
\frac{\Gamma_{W}^{\mathrm{osc}}}{H_{\mathrm{osc}}} \sim \frac{0.06 g^{2} m_{W}}{H_{\mathrm{osc}}} \sim 0.01\left(\frac{g}{0.5}\right)^{3}\left(\frac{0.01}{\lambda}\right)^{1 / 2} .
$$

We thus find that $\Gamma_{W}^{\text {osc }} \ll H_{\text {osc }}$ unless the higgs value during inflation would be extremely close to the instability point such that $\lambda \simeq 0$. The perturbative decay of the resonantly produced W-bosons can therefore be neglected at the beginning of the non-perturbative higgs decay. Similar arguments hold for Z-bosons and top quarks produced by the resonant higgs decay. Note that this is different from the decay of the non-minimally coupled higgs field after the end of higgs inflation. In that case the large higgs amplitude renders the perturbative decay channels of the higgs decay products important which affects the initial stages of the non-perturbative decay [19].

\subsection{Resonant production of gauge bosons and higgs particles}

We start by considering the non-perturbative production of weak gauge bosons by the resonant decay of the higgs condensate. For simplicity we will neglect the nonAbelian self-couplings of the gauge bosons. This should not generate significant errors during the first stages of the resonance when the number densities are still small.

In the unitarity gauge the equation of motion for the transverse components of the rescaled $\mathcal{W}_{\mu}^{ \pm}=a^{3 / 2} W_{\mu}^{ \pm}$gauge bosons is given by

$$
\ddot{\mathcal{W}}_{\mu}^{ \pm}(z, k)+\omega_{k}^{2} \mathcal{W}_{\mu}^{ \pm}(z, k)=0, \quad \omega_{k}^{2}=\frac{k^{2}}{a^{2} \lambda h_{\mathrm{osc}}^{2}}+q_{W} \frac{h(z)^{2}}{h_{\mathrm{osc}}^{2}}+\Delta .
$$

Here the resonance parameter is defined as $q_{W}=m_{W}(x)^{2} /\left(\lambda h(x)^{2}\right)=g^{2} /(4 \lambda)$ and $\Delta=-(3 / 4)(\dot{a} / a)^{2}-(3 / 2) \ddot{a} / a$. For the matter dominated background we have $\Delta=0$. Here use a re-scaled cosmic time variable $z=\sqrt{\lambda h_{\mathrm{osc}}^{2}}\left(t-t_{\mathrm{osc}}\right)$ and the over dot denotes a derivative with respect to $z$.

The equation of motion for the $Z$ bosons takes the same form (4.2) but the resonance parameter $q_{W}$ gets replaced by $q_{Z}=\left(g^{2}+g^{\prime 2}\right) /(4 \lambda)$. The decay of the 
higgs condensate into higgs particles is also controlled by an equation of the same form but with $q_{W}$ replaced by $q_{h}=m_{h}^{2} /\left(\lambda h^{2}\right)=3$.

Neglecting the backreaction of the resonantly generated particles, the dynamics of the higgs condensate is determined by

$$
\frac{\ddot{h}}{h_{\mathrm{osc}}}+3 \frac{H}{\sqrt{\lambda} h_{\mathrm{osc}}} \frac{\dot{h}}{h_{\mathrm{osc}}}+\left(\frac{h}{h_{\mathrm{osc}}}\right)^{3}=0,
$$

where we have neglected the subdominant bare mass term of the higgs field, $\sqrt{2 \lambda} v \propto$ $v$. According to Eqs. (2.3) and (2.6) we have $h_{\mathrm{osc}} \sim 0.1 \lambda_{*}^{1 / 4} H_{*}$. For $H_{*} \gtrsim 10^{6} \mathrm{GeV}$ we obtain $h_{\text {osc }} \gg v=246 \mathrm{GeV}$ and therefore the effect of the bare mass would be insignificant. For $H_{*} \simeq 10^{4} \mathrm{GeV}$ we have $h_{\mathrm{osc}} / v \sim \mathcal{O}(1)$ so in this case the bare mass could be of some importance. Nevertheless, we ignore the bare mass term here because other effects, such as the thermal mass of the higgs due to the produced SM particles [20], could be equally important. We leave elaborate consideration of these effects for later publications.

The induced gauge boson masses $m_{W}=g h / 2, m_{Z}=\sqrt{g^{2}+g^{\prime 2}} h / 2$ vanish each time when the oscillating higgs field crosses zero. The same also happens for the dominant contribution to the higgs mass, $3 \lambda h^{2}$, although the field does not become strictly massless due to the small bare mass term. During these short time intervals the effective masses change non-adiabatically $\left|\dot{m}_{W, Z, h}\right| / m_{W, Z, h}^{2} \gtrsim 1$ and a copious production of $W, Z$ and $h$ quanta can occur [16, 17].

The generation of the weak gauge bosons under the first stages of the resonance can be straightforwardly analysed by solving the equations of motion (4.2) and (4.3) numerically. From the mode functions we then obtain the corresponding number densities of the gauge bosons by using the relation [17]

$$
n_{k}=\frac{\omega_{k}}{2}\left(\frac{\left|\dot{\mathcal{W}}_{\mu}^{ \pm}\right|^{2}}{\omega_{k}^{2}}+\left|\mathcal{W}_{\mu}^{ \pm}\right|^{2}\right)-\frac{1}{2}
$$

The behaviour of the number density of $W$ or $Z$ bosons with $q=1.5$ and $k=0$ is illustrated in Fig. (1). The growth of the number density is approximatively exponential with ${ }^{2}$

$$
n_{k} \propto \exp \left(4.6 \mu_{k} z^{1 / 3}\right)
$$

where $\mu_{k}$ is a characteristic exponent. In Table (1) we show the numerically calculated characteristic exponents $\mu_{k}$ of zero-momentum quanta for four different choices of the inflationary scale, $H_{*} / \mathrm{GeV}=10^{4}, 10^{6}, 10^{8}, 10^{10}$, and both for the $W$ and $Z$ bosons. The inflationary scale $H_{*}$ sets the initial higgs value through equation (2.4) and thereby eventually determines the energy scale at which the Standard Model couplings are evaluated.

\footnotetext{
${ }^{2}$ In the conformal time, $x=\sqrt{\lambda h_{\text {osc }}^{2}}\left(\eta-\eta_{\text {osc }}\right)$, the number density evolves as $n_{k} \propto \exp \left(2 \mu_{k} x\right)$ $[17]$.
} 
The strength of the resonance is controlled by the resonance parameter $q=q_{W, Z, h}$ in equation (4.2). The resonance is typically stronger for larger values of $q$ but the relation is not monotonic and for some values the resonance will not take place at all. The structure of the resonance is closely related to that encountered in the conformally invariant scalar theory $V=\lambda \phi^{4} / 4+g^{2} \phi^{2} \chi^{2} / 2$ [17]. Indeed, apart from the small bare mass term which we have neglected and the matter dominated background in our case, the gauge field equation of motion (4.2) coincides with the conformally invariant form. For the conformal model, the zero-momentum quanta are resonantly amplified in the instability bands $q=n(n+1) / 2 \ldots(n+1)(n+2) / 2$, where $n$ is an odd integer [17]. Thus, the first three instability bands are $q=1 \ldots 3, q=6 \ldots 10$ and $q=15 \ldots 21$. These instability bands also seem to describe relatively well the resonance in our case. This can also be seen in Table (1) where the characteristic exponent $\mu_{k}$ is non-zero inside the instability bands and outside the bands it vanishes.

Usually quanta with low momenta are most efficiently produced and if particles with $k=0$ are not excited then the resonance is relatively weak. Thus, we consider here only the resonance bands corresponding to $k=0$. As the resonance parameters $q_{W}$ and $q_{Z}$ have a different dependence on the Standard Model couplings we find that for most of the parameter space at least one of the resonant decay channels into weak gauge bosons is efficient. It is however also possible that both resonance parameters happen to be outside the resonance bands. In such a situation the decay of the higgs condensate would be delayed.

For the decay of the higgs condensate into higgs particles the resonance parameter takes the value $q_{h}=3$. This lies on the edge of the instability band [17] and therefore we obtain an anomalously low characteristic exponent $\mu_{k} \approx 0.033$ for $k=0$. We thus find that the decay of the higgs condensate into higgs particles is generically subdominant compared to the decay into gauge bosons.

Table 1: Numerical values of the characteristic exponent $\mu_{k}$ of $k=0$ modes for a set of different values of $H_{*}$.

\begin{tabular}{cccc}
\hline \hline$H_{*} / \mathrm{GeV}$ & $\lambda$ & $\left(q_{W}, \mu_{k}\right)$ & $\left(q_{Z}, \mu_{k}\right)$ \\
\hline $10^{4}$ & 0.09 & $(1.1,0.14)$ & $(1.5,0.26)$ \\
$10^{6}$ & 0.04 & $(2.3,0.25)$ & $(3.2,0.00)$ \\
$10^{8}$ & 0.02 & $(4.4,0.00)$ & $(6.2,0.14)$ \\
$10^{10}$ & 0.005 & $(16,0.22)$ & $(24,0.00)$ \\
\hline
\end{tabular}

\subsection{Time scale of the higgs decay}

As the number of the produced gauge field quanta grows, they start to affect the dynamics of the oscillating higgs field and eventually shut down the resonance. The subsequent evolution towards thermal equilibrium is a highly complicated process 


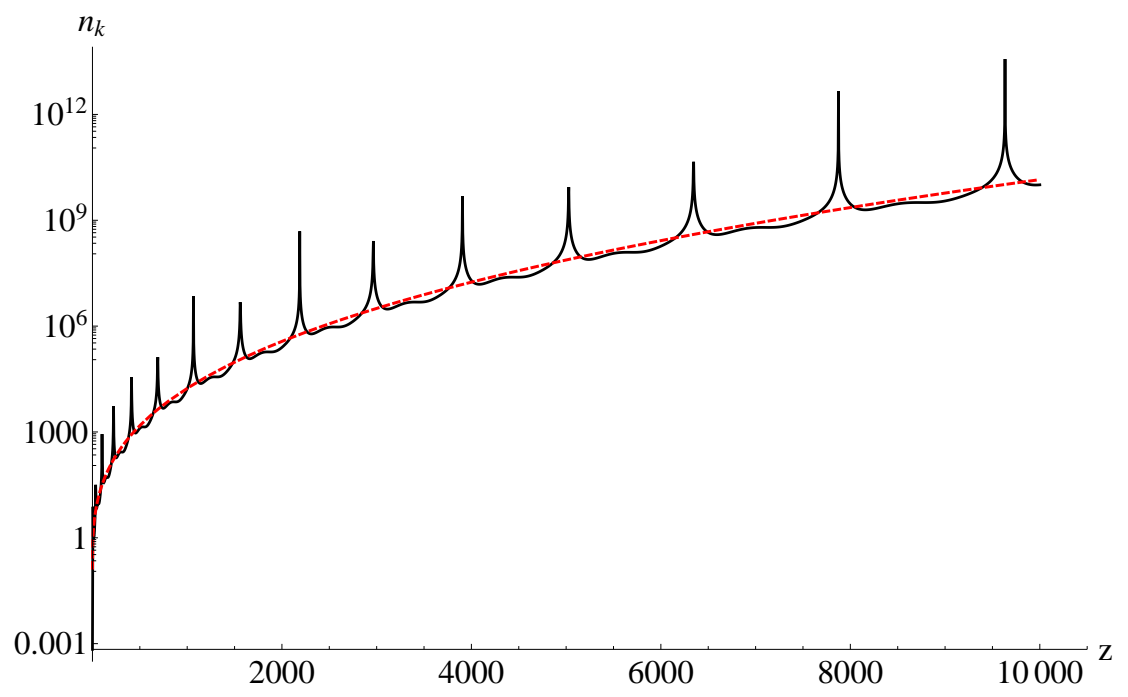

Figure 1: The evolution of the number density $n_{k}$ with $k=0$ and $q=1.5$ (black solid line) and exponential fitting (red dashed line) $n_{k} \propto \exp \left(4.6 \mu_{k} z^{1 / 3}\right)$, with $\mu_{k} \approx 0.26$.

which we do not aim to address here. Because of the non-linear dynamics of the later stages of the resonance, it is also a non-trivial task to precisely determine the time when the higgs condensate has decayed. For our purposes it suffices to estimate the decay time by the time when the induced higgs mass $m_{h(W, Z)}^{2}$ generated by the excited gauge bosons becomes equal to the mass term $m_{h}^{2}=3 \lambda h^{2}$. At this stage the backreaction becomes significant and our analysis can no longer be applied to describe the system.

The higgs coupling to weak gauge bosons in the unitarity gauge takes at large field values $h \gg v$ the form

$$
\mathcal{L}_{\text {int }}=q_{W} \lambda h^{2} W^{\mu+} W_{\mu}^{-}+\frac{1}{2} q_{Z} \lambda h^{2} Z^{\mu} Z_{\mu}
$$

where $q_{W}=g^{2} /(4 \lambda)$ and $q_{Z}=\left(g^{2}+g^{\prime 2}\right) /(4 \lambda)$ as before. The effective higgs mass generated by the $W_{\mu}^{ \pm}$bosons is then given by

$$
m_{h(W)}^{2}=2 q_{W} \lambda\left\langle W^{\mu+} W_{\mu}^{-}\right\rangle=2 q_{W} \lambda\left\langle W^{2}\right\rangle
$$

and the mass generated by $Z_{\mu}$ is obtained by replacing $2 q_{W}$ by $q_{Z}$. The resonantly produced higgs particles also contribute to the effective mass of the condensate but this is a subdominant effect as the higgs quanta are produced at a much lower rate than gauge bosons.

The expectation value in equation (4.7) can be approximated as [17]

$$
\left\langle W^{2}\right\rangle \approx \frac{1}{(2 \pi)^{3}} \int \frac{d^{3} k n_{k}}{\sqrt{k^{2}+q_{W} \lambda h_{\mathrm{osc}}^{2}}},
$$


where we have normalized the scale factor to unity at the onset of higgs oscillations $a_{\text {osc }}=1$. Only gauge bosons with rather low momenta are produced, so we can approximate $k^{2}+q_{W} \lambda h_{\mathrm{osc}}^{2} \approx q_{W} \lambda h_{\mathrm{osc}}^{2}$. For the number density of produced particles we use the fitting $n_{k} \propto \exp \left(4.6 \mu_{k} z^{1 / 3}\right)$. To obtain a crude estimate, we approximate the occupation numbers by $n_{k} \approx n_{k=0}$ up to the cutoff scale $k_{*} \approx\left(\frac{q_{W}}{2 \pi^{2}}\right)^{1 / 4} \sqrt{\lambda} h_{\text {osc }}$ [17] and set $n_{k}=0$ above this scale. This yields the estimate

$$
\left\langle W^{2}\right\rangle \approx \frac{n_{k}}{(2 \pi)^{3} \sqrt{q_{W} \lambda h_{\mathrm{osc}}^{2}}} \frac{4 \pi}{3} k_{*}^{3} \approx 1.8 \cdot 10^{-3} q_{W}^{1 / 4} \lambda h_{\mathrm{osc}}^{2} n_{k}
$$

We approximate the time scale of the higgs decay by the time when the backreaction of the decay products becomes significant $m_{h(W)}^{2} \approx m_{h}^{2}$, which occurs at

$$
n_{k} \sim \frac{1.3 \cdot 10^{3}}{2 q_{W}^{5 / 4} \lambda z_{\mathrm{dec}}^{2 / 3}},
$$

where we have make use of the fact that the scale factor in the matter dominated background is given by $a \approx(3 z / 2)^{2 / 3}$ for $z \gg 1$. From Eq. (4.10) with $n_{k} \propto$ $\exp \left(4.6 \mu_{k} z^{1 / 3}\right)$ the time of higgs decay $z_{\mathrm{dec}}$ can be solved. As $t_{\mathrm{dec}}=z_{\mathrm{dec}} H_{\mathrm{osc}}^{-1}$ for $t \gg t_{\mathrm{osc}}$, the value $z_{\mathrm{dec}}$ directly gives the number of Hubble times from the onset of higgs oscillations to the decay of the higgs condensate. In (4.10) we have for simplicity assumed the production of $W$ gauge bosons dominates over the $Z$ boson production. If the production of $Z$ gauge bosons is dominant $q_{W}^{5 / 4}$ in (4.10) should be replaced by $q_{Z}^{5 / 4} / 2$.

Table 2: The amount of Hubble times $H_{\mathrm{osc}} / H_{\mathrm{dec}}$ from the onset of higgs oscillations to the higgs decay. The number of inflaton oscillations $n_{\phi}^{\text {dec }}$ from the end of inflation until the higgs decay is also shown.

\begin{tabular}{cccc}
\hline \hline$H_{*} / \mathrm{GeV}$ & $\lambda$ & $H_{\text {osc }} / H_{\text {dec }}$ & $n_{\phi}^{\text {dec }}$ \\
\hline $10^{4}$ & 0.09 & 370 & 1000 \\
$10^{6}$ & 0.04 & 360 & 1700 \\
$10^{8}$ & 0.02 & 630 & 5100 \\
$10^{10}$ & 0.005 & 340 & 7700 \\
\hline
\end{tabular}

While equation (4.10) admits a general analytical solution, we will here only list its solutions for a set of parameter values. The results are shown Table in (2) which shows that the higgs condensate typically decays in a time scale of a few hundred of Hubble times after the onset of oscillations, $H_{\mathrm{osc}} / H_{\mathrm{dec}}=\mathcal{O}\left(10^{2}\right)$. We have also listed the corresponding number of inflaton oscillations measured from the end of inflation

$$
n_{\phi}^{\mathrm{dec}}=\frac{m_{\phi} t_{\mathrm{dec}}}{2 \pi} \sim \frac{1}{2 \pi} \frac{t_{\mathrm{dec}}}{H_{*}^{-1}} .
$$


Comparing with the results of Sect. 3, we find that the non-perturbative decay occurs way before the perturbative decay channels would become efficient. Looking at the cases shown in Table (2) we observe that decay is slowest for $H_{*}=10^{8} \mathrm{GeV}$ for which one of the resonance parameters is outside the resonance bands and the other one happens to be on the edge of a resonance band. Even in this case the higgs decay through the non-perturbative channels occurs much before the perturbative channels become relevant.

\subsection{Resonant production of quarks and leptons}

The Standard Model higgs $(h \gg v)$ couples to quarks and leptons in the unitarity gauge according to

$$
\mathcal{L}_{\text {int }}=-\sqrt{q_{i} \lambda} h f \bar{f}
$$

where $q_{i}=\frac{m_{i}^{2}}{\lambda h^{2}}=\frac{y_{i}^{2}}{2 \lambda}$. As the oscillating higgs field crosses zero fermions can be resonantly produced. Due to the fermion statistics, the generated fermions can populate states only up to the fermisphere whose radius is determined by the width of the resonance and grows along with the expansion of space [18]. The number density of fermions generated by the higgs decay therefore cannot compete with the exponentially growing number of gauge bosons produced by the bosonic resonance channels.

Since $\mathcal{L}_{\text {int }} \propto h$ the fermions do not give rise to effective mass for the higgs condensate at tree level contrary to the gauge bosons. However, the fermions do change the equation of motion of the higgs field (4.3) via backreaction. In the Hartree approximation one finds

$$
\frac{\ddot{h}}{h_{\mathrm{osc}}}+3 \frac{H}{\sqrt{\lambda} h_{\mathrm{osc}}} \frac{\dot{h}}{h_{\mathrm{osc}}}+\left(\frac{h}{h_{\mathrm{osc}}}\right)^{3}+\frac{\sqrt{q_{i}}}{\sqrt{\lambda} h_{\mathrm{osc}}^{3}}\langle f \bar{f}\rangle=0
$$

from which one could examine the backreaction effects of the generated fermions and the associated time scales, see [21]. However, as the number density of the resonantly generated gauge bosons grows exponentially as opposed to the fermions, it is clear that the dominant decay channels of the higgs condensate are the bosonic ones. For our purposes, the fermionic channels are therefore irrelevant and we will not address the details of the resonant fermion production further.

\section{Discussion}

The remarkable detection of a new boson consistent with the Standard Model higgs [1] by the ATLAS and CMS collaborations also suggest that during the inflationary epoch there was at least one other scalar in addition to the inflaton field. The minimal scenario where the higgs itself would be the inflaton [2] unfortunately requires a large 
non-minimal coupling to gravity and therefore does not seem to represent the generic situation.

In the present paper we have explored the fate of the minimally coupled Standard Model higgs during and after inflation assuming the Standard Model remains valid up to inflationary energy scales. The effective higgs potential is then exactly calculable in terms of the measured Standard Model parameters. We have shown that for any initial higgs value in the range where the Standard Model vacuum is stable, the expectation value of the higgs mass becomes much smaller than the Hubble scale over a period of less than about 30 efolds of inflation. The higgs then generically corresponds to a light spectator field during inflation and it acquires a spectrum of nearly scale invariant perturbations over the observable scales. The distribution of the higgs field relaxes to an equilibrium form with a calculable variance in between 20 and 85 efoldings, depending on the inflationary scale $H_{*}$.

After the end of inflation the higgs becomes effectively massive and starts to oscillate in less than $\mathcal{O}\left(10^{2}\right)$ Hubble times. We find that the oscillating higgs condensate generically decays into weak gauge bosons through a parametric resonance within a few hundreds of Hubble times from the onset of oscillations. The precise number depends on the inflationary scale $H_{*}$ as shown in Table 2.

At the time of inflation the higgs condensate contributes very little to the total energy density and its perturbations amount to a small isocurvature component. The higgs perturbations could however be a source of significant metric perturbations at a later stage if the expansion history after inflation is sensitive to the exact value of the higgs condensate. This could happen for example through the modulated reheating mechanism with the higgs modulating the inflaton decay $[9,11,5]$. Whether such a conversion takes place depends on details of the inflaton sector beyond Standard Model which we have not specified here. However, a general necessary condition for the scenario is that the inflaton decay should occur before the decay of the higgs condensate. As we have shown in this work, the decay of the Standard Model higgs takes place after $n_{\phi}=\mathcal{O}\left(10^{3}\right)$ oscillations of the inflaton field. A necessary condition for generating significant perturbations through a modulated reheating scenario with the higgs therefore is that the inflaton couplings should be large enough for it to decay within this time. Similar remarks can be made on the curvaton scenario with the Standard Model higgs although the viability of the mechanism is hampered by the quartic form of the higgs potential [5].

As the Standard Model higgs generically acquires perturbations during inflation it would be interesting to specify the general conditions under which its perturbations can significantly contribute to the observable primordial perturbations. Our findings suggest that curvaton-like conversion mechanisms, where the inflaton has to decay before the higgs, imply relatively strong couplings on the inflaton sector to be efficient. If the inflaton decays directly into Standard Model degrees of freedom it would be interesting to investigate if the required strength of the couplings is con- 
sistent with assuming negligible modifications to the effective potential for Standard Model fields from the inflaton sector. We plan to address these topics more carefully in a future work.

\section{Acknowledgments}

KE is supported by the Academy of Finland grants 1263714 and 1218322 . TM is supported by the Magnus Ehrnrooth foundation. SN is supported by the Academy of Finland grant 257532.

\section{References}

[1] G. Aad et al. [ATLAS Collaboration], Phys. Lett. B 716 (2012) 1 [arXiv:1207.7214 [hep-ex]]; S. Chatrchyan et al. [CMS Collaboration], Phys. Lett. B 716 (2012) 30 [arXiv:1207.7235 [hep-ex]].

[2] F. L. Bezrukov and M. Shaposhnikov, Phys. Lett. B 659, 703 (2008) [arXiv:0710.3755 [hep-th]]; A. De Simone, M. P. Hertzberg and F. Wilczek, Phys. Lett. B 678, 1 (2009) [arXiv:0812.4946 [hep-ph]].

[3] C. P. Burgess, H. M. Lee and M. Trott, JHEP 1007, 007 (2010) [arXiv:1002.2730 [hep-ph]]; C. P. Burgess, H. M. Lee and M. Trott, JHEP 0909, 103 (2009) [arXiv:0902.4465 [hep-ph]].

[4] K. Enqvist and M. S. Sloth, Nucl. Phys. B 626 (2002) 395 [arXiv:hep-ph/0109214]. D. H. Lyth and D. Wands, Phys. Lett. B 524, 5 (2002) [arXiv:hep-ph/0110002]. T. Moroi and T. Takahashi, Phys. Lett. B 522, 215 (2001) [Erratum-ibid. B 539, 303 (2002)] [arXiv:hep-ph/0110096]; A. D. Linde and V. F. Mukhanov, Phys. Rev. D 56 (1997) 535 [arXiv:astro-ph/9610219]; S. Mollerach, Phys. Rev. D 42 (1990) 313.

[5] K. -Y. Choi and Q. -G. Huang, arXiv:1209.2277 [hep-ph].

[6] T. Kunimitsu and J. Yokoyama, Phys. Rev. D 86 (2012) 083541 [arXiv:1208.2316 [hep-ph]].

[7] D. H. Lyth, JCAP 0511, 006 (2005) [astro-ph/0510443].

[8] G. Dvali, A. Gruzinov and M. Zaldarriaga, Phys. Rev. D 69, 023505 (2004) [arXiv:astro-ph/0303591]; L. Kofman, arXiv:astro-ph/0303614.

[9] A. De Simone and A. Riotto, JCAP 1302, 014 (2013) [arXiv:1208.1344 [hep-ph]].

[10] P. A. R. Ade et al. [Planck Collaboration], arXiv:1303.5084 [astro-ph.CO].

[11] A. De Simone, H. Perrier and A. Riotto, JCAP 1301, 037 (2013) [arXiv:1210.6618 [hep-ph]]. 
[12] G. Degrassi, S. Di Vita, J. Elias-Miro, J. R. Espinosa, G. F. Giudice, G. Isidori and A. Strumia, JHEP 1208 (2012) 098 [arXiv:1205.6497 [hep-ph]].

[13] A. A. Starobinsky, Lect. Notes in Phys., v. 246, pp. 107-126 (1986);

A. A. Starobinsky and J. Yokoyama, Phys. Rev. D 50, 6357 (1994) [astro-ph/9407016].

[14] K. Enqvist, R. N. Lerner, O. Taanila and A. Tranberg, JCAP 1210 (2012) 052 [arXiv:1205.5446 [astro-ph.CO]].

[15] A. Djouadi, Phys.Rept.457:1-216,2008 [arXiv:hep-ph/0503172].

[16] L. Kofman, A. D. Linde and A. A. Starobinsky, Phys. Rev. D 56 (1997) 3258 [hep-ph/9704452].

[17] P. Greene, L. Kofman, A. Linde, A. Starobinsky Phys.Rev.D56:6175-6192,1997 [arXiv:hep-ph/9705347].

[18] P. B. Greene, L. Kofman Phys.Lett.B448:6-12,1999 [arXiv:hep-ph/9807339];

J. Baacke, K. Heitmann and C. Patzold, Phys. Rev. D 58 (1998) 125013 [hep-ph/9806205]; P. B. Greene and L. Kofman, Phys. Rev. D 62 (2000) 123516 [hep-ph/0003018].

[19] J. Garcia-Bellido, D. G. Figueroa and J. Rubio, Phys. Rev. D 79 (2009) 063531 [arXiv:0812.4624 [hep-ph]]; F. Bezrukov, D. Gorbunov and M. Shaposhnikov, JCAP 0906 (2009) 029 [arXiv:0812.3622 [hep-ph]].

[20] K. Enqvist, D. G. Figueroa and R. N. Lerner JCAP 1301 (2013) 040 [arXiv:1211.5028 [astro-ph.CO]].

[21] G. F. Giudice, M. Peloso, A. Riotto and I. Tkachev JHEP 9908 (1999) 014 [arXiv:hep-ph/9905242]. 